\title{
MATHEMATICAL ANALYSIS OF A SPECTRAL HYPERVISCOSITY LES MODEL FOR THE SIMULATION OF TURBULENT FLOWS
}

\author{
JeAn-LuC Guermond $^{1,2}$ And Serge Prudhomme ${ }^{2,3}$
}

\begin{abstract}
This paper presents a model based on spectral hyperviscosity for the simulation of 3D turbulent incompressible flows. One particularity of this model is that the hyperviscosity is active only at the short velocity scales, a feature which is reminiscent of Large Eddy Simulation models. We propose a Fourier-Galerkin approximation of the perturbed Navier-Stokes equations and we show that, as the cutoff wavenumber goes to infinity, the solution of the model converges (up to subsequences) to a weak solution which is dissipative in the sense defined by Duchon and Robert (2000).
\end{abstract}

Mathematics Subject Classification. 35Q30, 65N35, 76M05.

Received: March 27, 2003. Revised: July 1, 2003.

\section{INTRODUCTION}

\subsection{Dissipative and suitable weak solutions}

It is generally accepted that the Navier-Stokes equations stand as a reasonable model to predict the behavior of turbulent incompressible flows of viscous fluids. Upon denoting by $\Omega$ the domain occupied by the fluid, $[0, T]$ some time interval, $u$ the velocity field, and $p$ the pressure, the problem is formulated as follows:

$$
\left\{\begin{array}{l}
\partial_{t} u+u \cdot \nabla u+\nabla p-\nu \Delta u=f \text { in } Q_{T}, \\
\nabla \cdot u=0 \text { in } Q_{T} \\
\left.u\right|_{\Gamma}=0 \text { or } u \text { is periodic, } \\
\left.u\right|_{t=0}=u_{0}
\end{array}\right.
$$

where $Q_{T}=\Omega \times(0, T), \Gamma$ is the boundary of $\Omega, u_{0}$ the solenoidal initial data, $f$ a source term, $\nu$ the kinematic viscosity, and the density is chosen equal to unity.

In mathematical terms, the turbulence question is an elusive one. Since the bold definition of turbulence by Leray in the 1930's [20], calling solution turbulente any weak solution of the Navier-Stokes equations, progress has been frustratingly slow. The major obstacle in analyzing the Navier-Stokes equations has to do with the

\footnotetext{
Keywords and phrases. Navier-Stokes equations, turbulence, large Eddy simulation.

1 LIMSI (CNRS-UPR 3152), BP 133, 91403, Orsay, France. e-mail: guermond@limsi.fr

2 ICES, formerly TICAM, The University of Texas at Austin, TX 78712, USA

3 On leave at Universidad de los Andes, Bogotá, Colombia. e-mail: serge@ices.utexas.edu
} 
question of uniqueness of solutions in 3D, a question not yet solved owing to the possibility that the occurrence of so-called vorticity bursts reaching scales smaller than the Kolmogorov scale cannot be excluded.

If weak solutions are not unique, a fundamental question is then to distinguish the physically relevant solutions. A possible piece of the puzzle may have been found by Scheffer [27], Caffarelli et al. [4], and Duchon and Robert [10] who introduced the notion of "dissipative solutions" or "suitable weak solutions:"

Definition 1.1. A weak solution to the Navier-Stokes equation is dissipative (or suitable) if it is in the class $L^{2}\left(0, T ; H^{1}(\Omega)\right) \cap L^{\infty}\left(0, T ; L^{2}(\Omega)\right)$ and is such that the local energy balance

$$
\partial_{t}\left(\frac{1}{2} u^{2}\right)+\nabla \cdot\left(\left(\frac{1}{2} u^{2}+p\right) u\right)-\nu \Delta\left(\frac{1}{2} u^{2}\right)+\nu(\nabla u)^{2}-f \cdot u \leq 0
$$

is satisfied in the sense of distributions.

It is remarkable that, to date, it is for the class of "suitable weak solutions" that the best partial regularity result has been proved (see Caffarelli et al. [4] and Scheffer [27]); that is, the one-dimensional Hausdorff measure of singular points of such solutions is zero. In other words, dissipative solutions (or suitable weak solutions) are almost classical (i.e., smooth). Whether these solutions are indeed classical is still far from being clear (see e.g. Scheffer [28]).

Moreover, Duchon and Robert have given an explicit form of the distribution, $D(u)$, that is missing in the left-hand side of (1.2) to reach equality. For a smooth flow, the distribution in question is zero; but for nonregular flow it may be nontrivial. Dissipative solutions are those such that $D(u) \geq 0$. As pointed out by Duchon and Robert, it is remarkable that the solutions turbulentes of Leray are dissipative.

Since it is not yet known whether weak solutions obtained as limits of Galerkin approximations are dissipative (or suitable), the principle driving our line of thoughts in the present paper is to conjecture that, indeed, it is not the case, and that (1.2) is a reasonable criterion for selecting almost classical solutions.

\subsection{Large Eddy Simulations}

In recent years, significant progress toward the development of turbulent models has occurred based on the idea that the whole range of flow scales may not be important in many significant engineering applications. This has led modelers to devise artifacts for representing the interaction between the unreachable small scales and the large ones. These models are commonly known as Large Eddy Simulation (LES) models, an acronym coined in the ground breaking paper of Leonard [19]. The primary goal of LES is to modify the Navier-Stokes equations in order to obtain a new system of equations which is more amenable to approximation while retaining all the most energetic features of the unperturbed problem. Many LES models have been proposed, but no satisfactory mathematical theory for LES has yet emerged.

The authors have recently attempted to analyze Large Eddy Simulation models from a mathematical perspective, [11]. This work lead us to suggest some criteria for developing a rigorous mathematical theory of LES. We proposed that a LES model should regularize the Navier-Stokes equations and should be able to select physically relevant solutions like dissipative solutions à la Duchon-Robert. For instance, Leray's regularization satisfies these two criteria. As a matter of fact, Leray's mollification is at the origin of the so-called NS- $\alpha$ model [6]. The nonlinear viscosity models of Ladyženskaja, Lions, and Kaniel $[12,16,17,23]$ also comply with the proposed criteria. Among the admissible nonlinear viscosity is the $p$-Laplacian provided $p-2>\frac{1}{2}$. Since Smagorinsky's model [29] is a 3-Laplacian, this model selects dissipative solutions. This fact may be one of the reasons, among possibly many, explaining the effectiveness of the Smagorinsky model and its many variants frequently reported in the literature, see e.g. [8]. On the other hand, LES methods based on spectral approximations generally fall short of complying with these two criteria. The spectral viscosity model of Tadmor $[5,25,30]$, which was originally developed for nonlinear scalar conservation laws, and later extended to large eddy simulation of viscous flows by Karamanos and Karniadakis [13] or Adams and Stolz [1], may not yield a dissipative solution. Other techniques such as the spectral eddy-viscosity methods developed by Kraichnan [15], Chollet and Lesieur [7], Lamballais, Métais, and Lesieur [18], Lesieur and Roggalo [21], or McComb and Young [26], 
generally lose spectral accuracy when the flow is eventually resolved and does not guarantee convergence to a dissipative solution when the cutoff goes to infinity. As an alternative to these methods, the goal of the present paper is to present a new spectral hyperviscosity model, following an approach proposed by Lions [22,24], and to show that this technique preserves spectral accuracy and guarantees convergence to a dissipative solution.

\subsection{Organization of the paper}

The paper is organized as follows. In Section 2 we introduce Lions' hyperviscosity model and briefly recall important results. In essence this type of viscosity regularizes the Navier-Stokes equations and selects dissipative solutions provided the exponent of the hyperviscosity is larger than $\frac{5}{4}$. In Section 3 we introduce a fully discrete spectral approximation of the Navier-Stokes equations, and we show how to tune the hyperviscosity model so that it introduces the least possible dissipation (i.e. it remains spectrally consistent) while ensuring the limit solution to be dissipative. The two main results of the paper are embodied in Theorem 4.1 and Theorem 5.1, which are proved in Section 4 and in Section 5, respectively. Concluding remarks follow in Section 6.

\subsection{Notations and conventions}

As usual, we denote by $H^{m}(\Omega)$ the Sobolev spaces of functions in $L^{2}(\Omega)$ with partial derivatives of order up to $m$ in $L^{2}(\Omega)$. We do not make notational distinctions between vector- and scalar-valued functions. Given a real number $p, 1 \leq p \leq \infty$, we denote by $p^{\prime}$ its conjugate such that $\frac{1}{p}+\frac{1}{p^{\prime}}=1$.

In the following, $A \lesssim B$ means that $A \leq c B$ where $c$ is a generic constant which may depend on the data $f$, $u_{0}, \nu, \Omega$. In Section 2 the generic constant may also depend on the parameter $\epsilon$, but in the rest of the paper this constant does not depend on the discretization parameters, namely $N$ and $\epsilon_{N}$. That is, all the estimates derived in Section 3 and further are uniform with respect to the discretization parameters.

\section{THE HYPERVISCOSITY MODEL}

The hyperviscosity model proposed by Lions $[22,24]$ consists in perturbing the Navier-Stokes equations as follows

$$
\left\{\begin{array}{l}
\partial_{t} u+u \cdot \nabla u+\nabla p-\nu \Delta u+\epsilon(-\Delta)^{m} u=f \text { in } Q_{T}, \\
\nabla \cdot u=0 \quad \text { in } Q_{T}, \\
u, \frac{\partial u}{\partial n}, \ldots, \frac{\partial^{m-1} u}{\partial n^{m-1}}=0 \quad \text { or } u \text { is periodic, on } \Gamma, \\
\left.u\right|_{t=0}=u_{0},
\end{array}\right.
$$

where $\epsilon>0$. The appealing aspect of this perturbation is that it yields a wellposed problem in the standard sense when $m \geq \frac{5}{4}$ in three space dimensions. More precisely, upon denoting by $d \geq 2$ the space dimension, we have the following result.

Theorem 2.1 (Lions [24]). Assume $f \in L^{2}\left(0, T ; L^{2}(\Omega)\right.$ ) and $u_{0} \in H^{m}(\Omega)$. Problem (2.1) has a unique solution in $L^{\infty}\left(0, T ; H^{m}(\Omega)\right)$ for all times $T>0$ if $m \geq \frac{d+2}{4}$.

Proof. We review here the main arguments of the proof as they will be subsequently referred to in the paper. Testing the momentum equation by $u$ yields

$$
\frac{1}{2} \frac{\mathrm{d}}{\mathrm{d} t}\|u\|_{L^{2}}^{2}+\nu\|\nabla u\|_{L^{2}}^{2}+\epsilon \sum_{[\alpha]=m}\left\|D^{\alpha} u\right\|_{L^{2}}^{2}=(f, u)
$$

which, after simplification and integration in time, gives the following estimate:

$$
\|u\|_{L^{2}}^{2}+\int_{0}^{T}\|u\|_{H^{m}}^{2} \mathrm{~d} t \lesssim\left\|u_{0}\right\|_{L^{2}}^{2}+\|f\|_{L^{2}\left(L^{2}\right)}^{2} .
$$


In a similar manner, testing by $u_{t}$ yields

$$
\left\|u_{t}\right\|_{L^{2}\left(L^{2}\right)}^{2}+\|u\|_{H^{m}}^{2} \lesssim\left\|u_{0}\right\|_{H^{m}}^{2}+\int_{0}^{T} \mathrm{~d} t \int_{\Omega}|u|^{2}|\nabla u|^{2} \mathrm{~d} x+\|f\|_{L^{2}\left(L^{2}\right)}^{2} .
$$

Using Hölder's inequality, we may write

$$
\int_{\Omega}|u|^{2}|\nabla u|^{2} \mathrm{~d} x \lesssim\|u\|_{L^{2 p}}^{2}\|\nabla u\|_{L^{2 p^{\prime}}}^{2}
$$

Moreover, owing to Sobolev inequalities we also have

$$
\begin{aligned}
& \|u\|_{L^{2 p}} \lesssim\|\nabla u\|_{L^{2 p^{\prime}}} \quad \text { if } \quad \frac{1}{2 p} \geq \frac{1}{2 p^{\prime}}-\frac{1}{d}, \\
& \|\nabla u\|_{L^{2 p^{\prime}}} \lesssim\|u\|_{H^{m}} \quad \text { if } \quad \frac{1}{2 p^{\prime}} \geq \frac{1}{2}-\frac{m-1}{d} .
\end{aligned}
$$

These two conditions yield

$$
m \geq \frac{d+2}{4} \quad \text { and } \quad p^{\prime} \geq \frac{2 d}{d+2}
$$

and

$$
\left\|u_{t}\right\|_{L^{2}\left(L^{2}\right)}^{2}+\|u\|_{H^{m}}^{2} \lesssim\left\|u_{0}\right\|_{H^{m}}^{2}+\int_{0}^{T}\|u\|_{H^{m}}^{2}\|u\|_{H^{m}}^{2} \mathrm{~d} t+\|f\|_{L^{2}\left(L^{2}\right)}^{2}
$$

and since $\int_{0}^{T}\|u\|_{H^{m}}^{2} \mathrm{~d} t$ is bounded (from estimate (2.3)), Gronwall's lemma yields

$$
\left\|u_{t}\right\|_{L^{2}\left(L^{2}\right)}+\|u\|_{L^{\infty}\left(H^{m}\right)} \lesssim c\left(\nu, u_{0}, f, \epsilon\right) .
$$

Existence of solutions in the class $L^{\infty}\left(0, T ; H^{m}\right)$, for all $T>0$, is then proved by means of the Galerkin technique using the a priori estimates (2.3)-(2.5) (see Lions [24]).

Uniqueness of solutions is shown as follows. Let $u_{1}$ and $u_{2}$ be two solutions in the $L^{\infty}\left(0, T ; H^{m}(\Omega)\right)$ class, and let us set $u=u_{1}$ and $w=u_{2}-u_{1}$. We first look for an estimate on the nonlinear term. Upon denoting by $q$ a real number such that $1 \leq q \leq \infty$, we infer

$$
\int_{\Omega}(w \cdot \nabla) u \cdot w \mathrm{~d} x \lesssim\|w\|_{L^{2 q^{\prime}}}^{2}\|\nabla u\|_{L^{q}} .
$$

Then, assuming $m \geq(d+2) / 4$, and taking $p_{0}^{\prime}=2 d /(d+2)$, it follows that

$$
\frac{1}{2 p_{0}^{\prime}} \geq \frac{1}{2}-\frac{m-1}{d}
$$

Therefore, by selecting $q=2 p_{0}^{\prime}$, we have

$$
\|\nabla u\|_{L^{q}} \lesssim\|u\|_{H^{m}} .
$$

Moreover, with these values for $p_{0}^{\prime}$ and $q$, we can check that $q^{\prime}=2 p_{0} /\left(1+p_{0}\right) \leq p_{0}$. It follows that $2 \leq 2 q^{\prime} \leq 2 p_{0}$, so that we can use the interpolation inequality (see for example Brezis [3, p.57])

$$
\|w\|_{L^{2 q^{\prime}}} \lesssim\|w\|_{L^{2}}^{a}\|w\|_{L^{2 p_{0}}}^{1-a}, \quad \text { with } \quad \frac{1}{2 q^{\prime}}=\frac{a}{2}+\frac{1-a}{2 p_{0}} .
$$


A straightforward calculation yields the value $a=\frac{1}{2}$. Moreover, since for this particular choice of $p_{0}, H^{m}(\Omega) \subset$ $L^{2 p_{0}}(\Omega)$, i.e., $\|w\|_{L^{2 p_{0}}} \lesssim\|w\|_{H^{m}}$, we have

$$
\|w\|_{L^{2 q^{\prime}}}^{2} \lesssim\|w\|_{L^{2}}\|w\|_{L^{2 p_{0}}} \lesssim\|w\|_{L^{2}}\|w\|_{H^{m}} .
$$

Combining the previous results, we arrive at the estimate:

$$
\int_{\Omega}(w \cdot \nabla) u \cdot w \mathrm{~d} x \lesssim\|w\|_{L^{2}}\|w\|_{H^{m}}\|u\|_{H^{m}}
$$

Finally, after subtracting the two momentum equations satisfied by $u_{1}$ and $u_{2}$ respectively, multiplying the result by $w$, and using the fact that $u_{2}$ is solenoidal, we obtain

$$
\|w\|_{L^{2}}^{2}+\int_{0}^{T}\|w\|_{H^{m}}^{2} \mathrm{~d} t \lesssim \int_{0}^{T}\|w\|_{L^{2}}\|w\|_{H^{m}}\|u\|_{H^{m}} \mathrm{~d} t \lesssim \int_{0}^{T}\|w\|_{L^{2}}\|w\|_{H^{m}} \mathrm{~d} t .
$$

Gronwall's lemma yields $w=0$; in other words, $u_{2}=u_{1}$.

Corollary 2.1. Let $\left(u_{\epsilon}\right)$ denote a sequence of solutions to (2.1) as $\epsilon$ tends to zero. Under the assumptions of Theorem 2.1, there exist subsequences $\left(u_{\epsilon_{l}}\right)$ that converge to a weak solution $u$ of $(1.1)$, weakly in $L^{2}\left(0, T ; H^{1}\right)$ and strongly in $L^{2}\left(0, T ; L^{2}\right)$. In addition, $u$ is dissipative in the sense of Definition 1.1.

Proof. See the proof of Theorem 4.1 and of Theorem 5.1 where stronger results are proved.

Remark 2.1. The hyperviscosity model of Lions is a LES model commonly used in the numerical simulation of geophysical flows (see e.g. Basdevant et al. [2], Kevlahan and Farge [14]). Corollary 2.1 actually shows that the hyperviscosity model complies with our requirements that a LES model should be a regularization when the regularization parameter, $\epsilon$, is fixed (i.e. yields a unique classical solution), and should yield a dissipative solution as $\epsilon$ goes to zero (we refer the reader to [11] where this point of view is discussed in details).

In the next sections, we turn our attention to the numerical point of view. We want to approximate the solution of (2.1) by means of the Galerkin technique, and we want to tune the hyperviscosity model so as to introduce the least possible dissipation, while still complying with the requirement that the limit solution be dissipative when both the inverse of the cutoff wavenumber and the regularization parameter tend to zero. The main results are established in Theorem 4.1 and Theorem 5.1.

\section{A SPECTRAL HYPERViSCOSITY MODEL}

We propose in this section to construct a spectral hyperviscosity model based on the program described above. One particularity of the model is that the hyperviscosity must act only on the short scales of the velocity field. We will show that this hyperviscosity perturbation remains small when compared with the norm of the solution (see Prop. 3.1). Henceforth we assume that $\Omega$ is the 3D torus, $\Omega=(0,2 \pi)^{3}$, so that periodic boundary conditions are enforced. Moreover, we assume that $f$ and $u_{0}$ are of zero mean value and that $f$ is solenoidal. The latter assumption is not restrictive since any gradient component of $f$ can be added to the pressure.

\subsection{Preliminaries and Fourier approximations}

For any $z \in \mathbb{C}^{\ell}, 1 \leq \ell \leq 3$, we denote by $|z|$ the Euclidean norm and by $|z|_{\infty}$ the maximum norm. We denote by $\bar{z}$ the conjugate of $z$. Recall that Sobolev spaces $H^{s}(\Omega), s \geq 0$, can be equivalently defined in terms of Fourier series as follows

$$
H^{s}(\Omega)=\left\{u=\sum_{k \in \mathbb{Z}^{3}} u_{k} \mathrm{e}^{\mathrm{i} k \cdot x}, u_{k}=\bar{u}_{-k}, \sum_{k \in \mathbb{Z}^{3}}\left(1+|k|^{2}\right)^{s}\left|u_{k}\right|^{2}<+\infty\right\} .
$$


In other words, the set of trigonometric polynomials $\exp (\mathrm{i} k \cdot x), k \in \mathbb{Z}^{3}$, is complete and orthogonal in $H^{s}(\Omega)$ for all $s \geq 0$. The scalar product in $L^{2}(\Omega)$ is denoted by $(u, v)=(2 \pi)^{-3} \int_{\Omega} u \bar{v}$ and the dual of $H^{s}(\Omega)$ by $H^{-s}(\Omega)$. We introduce the closed subspace $\dot{H}^{s}(\Omega)$ of $H^{s}(\Omega)$ composed of the functions of zero mean value. We will make use of the following equivalent norms

$$
\|u\|_{\dot{H}^{s}}^{2}=\sum_{k \neq 0, k \in \mathbb{Z}^{3}}|k|^{2 s}\left|u_{k}\right|^{2} .
$$

Recall that the Poincaré inequality holds in $\dot{H}^{s}(\Omega)$.

Let $N$ be a positive integer, henceforth referred to as the cutoff wave number. We introduce the set of trigonometric polynomials of partial degree less than or equal to $N$ :

$$
\mathbb{P}_{N}=\left\{p(x)=\sum_{|k|_{\infty} \leq N} c_{k} \mathrm{e}^{\mathrm{i} k \cdot x}, c_{k}=\bar{c}_{-k}\right\}
$$

and denote by $\dot{\mathbb{P}}_{N}$ the subspace of $\mathbb{P}_{N}$ composed of the trigonometric polynomials of zero mean value. We also consider the truncation operator $P_{N}: H^{s}(\Omega) \longrightarrow \mathbb{P}_{N}$ such that

$$
H^{s}(\Omega) \ni \sum_{k \in \mathbb{Z}^{3}} v_{k} \mathrm{e}^{\mathrm{i} k \cdot x}=v \longmapsto P_{N} v=\sum_{|k|_{\infty} \leq N} v_{k} \mathrm{e}^{\mathrm{i} k \cdot x} \in \mathbb{P}_{N} .
$$

Important properties of this operator are listed below:

Lemma 3.1. $P_{N}$ satisfies the following properties:

1) $P_{N}$ is the restriction on $H^{s}(\Omega)$ of the $L^{2}$ projection onto $\mathbb{P}_{N}$.

2) $\forall s \geq 0,\left\|P_{N}\right\|_{\mathcal{L}\left(H^{s}(\Omega) ; H^{s}(\Omega)\right)} \leq 1$.

3) $P_{N}$ commutes with differentiation operators.

4) $\forall v \in H^{s}(\Omega), \forall \mu, 0 \leq \mu \leq s,\left\|v-P_{N} v\right\|_{H^{\mu}} \lesssim N^{\mu-s}\|v\|_{H^{s}}$.

5) $\forall v \in \mathbb{P}_{N}, \forall \mu, s, s \leq \mu,\left\|P_{N} v\right\|_{H^{\mu}} \lesssim N^{\mu-s}\|v\|_{H^{s}}$.

Finally, to approximate the velocity and the pressure fields we introduce the following finite-dimensional vector spaces:

$$
X_{N}=\dot{\mathbb{P}}_{N}^{3}, \quad \text { and } \quad M_{N}=\dot{\mathbb{P}}_{N} .
$$

\subsection{Definition of the model}

The construction of the hyperviscosity model involves the definition of a vanishing viscosity amplitude $\epsilon_{N}$ and a hyperviscosity kernel $Q(x)$. We choose to construct the kernel in such a way that it acts only on the high wave numbers of the velocity field, namely $N_{i} \leq|k|_{\infty} \leq N, N_{i}$ standing for an intermediate cutoff wave number. This idea is quite similar to the spectral viscosity technique that Tadmor $[5,25,30]$ developed for nonlinear scalar conservation laws.

We start by introducing the hyperviscosity exponent $\alpha$, which will play a role similar to $m$ in the hyperviscosity model of Lions. We assume

$$
\alpha>\frac{5}{4}
$$


TABLE 1. Admissible values of the parameters $\alpha, \beta$, and $\theta$.

\begin{tabular}{|c|c|c|c|c|c|}
\hline$\alpha$ & $\frac{3}{2}$ & 2 & 3 & 4 & 5 \\
\hline$\beta$ & $<\frac{1}{2}$ & $<\frac{8}{7}$ & $<\frac{8}{3}$ & $<\frac{48}{11}$ & $<\frac{80}{13}$ \\
\hline$\theta$ & $<\frac{1}{6}$ & $<\frac{2}{7}$ & $<\frac{4}{9}$ & $<\frac{6}{11}$ & $<\frac{8}{13}$ \\
\hline
\end{tabular}

Then, we define the vanishing viscosity amplitude $\epsilon_{N}$, the intermediate cutoff wave number $N_{i}$, and the hyperviscosity kernel $Q(x)$ as follows:

$$
\left\{\begin{array}{l}
\epsilon_{N}=N^{-\beta}, \quad \text { with } \quad 0<\beta<2 \alpha \\
N_{i}=N^{\theta}, \quad \text { with } \quad \theta=\frac{\beta}{2 \alpha}, \\
Q(x)=(2 \pi)^{-3} \quad \sum_{N_{i} \leq|k|_{\infty} \leq N}|k|^{2 \alpha} \mathrm{e}^{\mathrm{i} k \cdot x} .
\end{array}\right.
$$

Note that with these definitions, we have $\epsilon_{N}=N_{i}^{-2 \alpha}$. Furthermore, we assume that the parameter $\beta$ satisfies the following bounds:

$$
0<\beta< \begin{cases}\frac{4 \alpha-5}{2} & \text { If } \alpha \leq \frac{3}{2} \\ \frac{4 \alpha(\alpha-1)}{2 \alpha+3} & \text { Otherwise. }\end{cases}
$$

These conditions imply the following bounds on $\theta$ :

$$
0<\theta< \begin{cases}1-\frac{5}{4 \alpha} & \text { If } \alpha \leq \frac{3}{2} \\ 1-\frac{5}{2 \alpha+3} & \text { Otherwise }\end{cases}
$$

Remark 3.1. As $\beta$ increases, the vanishing viscosity amplitude decreases and the range of wave numbers in which the kernel $Q(x)$ is active shrinks. Some admissible values of the parameters $\alpha, \beta$, and $\theta$ are shown in Table 1. Proposition 3.1 shows that the resulting hyperviscosity perturbation is spectrally small.

Remark 3.2. In practice, one can also define the hyperviscosity kernel as follows:

$$
Q(x)=\frac{1}{(2 \pi)^{3}} \sum_{N_{i} \leq|k|_{\infty} \leq N} \hat{Q}_{|k|}|k|^{2 \alpha} \mathrm{e}^{\mathrm{i} k \cdot x},
$$

where the viscosity coefficients $\hat{Q}_{|k|}$ are such that

$$
\left|1-\hat{Q}_{|k|}\right| \lesssim \frac{N_{i}^{2 \alpha}}{|k|^{2 \alpha}}, \quad \forall|k|_{\infty} \geq N_{i}
$$

This definition has the practical advantage of ensuring a smooth transition of the viscosity coefficients across the threshold $N_{i}$. All the results of the paper hold also with this definition. 
The spectral hyperviscosity model consists of seeking Fourier-Galerkin approximations of (1.1) as follows:

$$
\left\{\begin{array}{c}
\text { Find } u_{N} \in \mathcal{C}^{1}\left([0, T] ; X_{N}\right) \text { and } p_{N} \in \mathcal{C}^{0}\left([0, T] ; M_{N}\right) \text { such that } \\
\left(\partial_{t} u_{N}, v\right)+\left(u_{N} \cdot \nabla u_{N}, v\right)-\left(p_{N}, \nabla \cdot v\right)+\nu\left(\nabla u_{N}, \nabla v\right) \\
\quad+\epsilon_{N}\left(Q * u_{N}, v\right)=(f, v), \quad \forall v \in X_{N}, \forall t \in(0, T], \\
\left(\nabla \cdot u_{N}, q\right)=0, \quad \forall q \in M_{N}, \forall t \in(0, T], \\
\left.u_{N}\right|_{t=0}=P_{N} u_{0}
\end{array}\right.
$$

where $Q * u_{N}$ denotes the convolution product:

$$
Q * u_{N}(x)=\int_{\Omega} u_{N}(y) Q(x-y) \mathrm{d} y=\sum_{N_{i} \leq|k|_{\infty} \leq N}|k|^{2 \alpha} u_{k} \mathrm{e}^{\mathrm{i} k \cdot x} .
$$

Remark 3.3. We observe that $u_{N}$ is solenoidal. Indeed, $\nabla \cdot u_{N}$ is in $M_{N}$ so that replacing $q$ by $\nabla \cdot u_{N}$ in (3.6) yields $\left\|\nabla \cdot u_{N}\right\|_{L^{2}}=0$, that is $\nabla \cdot u_{N}=0$.

Proposition 3.1. The hyperviscosity perturbation is spectrally small in the sense that

$$
\epsilon_{N}\left\|Q * u_{N}\right\|_{L^{2}} \lesssim N^{-\theta s}\left\|u_{N}\right\|_{H^{s}}, \quad \forall u_{N} \in H^{s}(\Omega), \quad \forall s \geq 2 \alpha .
$$

Proof. Starting from the definition of the convolution product, we infer

$$
\left\|Q * u_{N}\right\|_{L^{2}}^{2}=\sum_{N_{i} \leq|k|_{\infty} \leq N}|k|^{4 \alpha}\left|u_{k}\right|^{2} .
$$

Using the fact that $2 \alpha \leq s$ and that $N_{i} \leq|k|_{\infty} \leq|k|$, we have:

$$
|k|^{4 \alpha}=N_{i}^{4 \alpha}\left(\frac{|k|}{N_{i}}\right)^{4 \alpha} \leq N_{i}^{4 \alpha}\left(\frac{|k|}{N_{i}}\right)^{2 s}=N_{i}^{4 \alpha-2 s}|k|^{2 s} .
$$

Moreover, from the definition of $N_{i}$ and $\theta$, we have

$$
N_{i}^{4 \alpha-2 s}=N^{\theta(4 \alpha-2 s)}=N^{2(2 \alpha \theta-\theta s)}=N^{2 \beta-2 \theta s}
$$

so that

$$
\epsilon_{N}^{2}\left\|Q * u_{N}\right\|_{L^{2}}^{2} \leq N^{-2 \beta} N^{2 \beta-2 \theta s} \sum_{N_{i} \leq|k|_{\infty} \leq N}|k|^{2 s}\left|u_{k}\right|^{2} \leq N^{-2 \theta s}\left\|u_{N}\right\|_{H^{s}}^{2}
$$

which establishes the result stated in (3.8).

Remark 3.4. Note that for $s \geq 2 \alpha$, we have $\theta s \geq \beta$ and $N^{-\theta s} \leq N^{-\beta}$. Thus, as $N$ grows larger, the consistency error, $N^{-\theta s}$, is far smaller than $\epsilon_{N}$.

\section{Convergence to a Weak solution of the nse}

In this section we show that, up to subsequences, the solution of the spectral hyperviscosity model (3.6) converges to a weak solution of the Navier-Stokes equation as $N$ goes to infinity. This result is stated in the following theorem.

Theorem 4.1. Let $f \in L^{2}\left(0, T ; L^{2}(\Omega)\right)$ and $u_{0} \in H^{\alpha}(\Omega)$. Assuming that (3.2), (3.4), and (3.3) hold, the solution $u_{N}$ to (3.6) is such that subsequences of $\left(u_{N}\right)$ converge weakly in $L^{2}\left(0, T ; H^{1}\right)$ and strongly in any $L^{p}\left(0, T ; L^{q}\right)$ with $2 \leq q<6$ and $2 \leq p<\frac{4 q}{3(q-2)}$, to a weak solution of (1.1) as $N$ goes to infinity. 


\subsection{A priori estimates}

The proof of Theorem 4.1 relies on several a priori estimates that are presented in the following lemmas.

Lemma 4.1. Under the assumptions of Theorem 4.1, the solution to (3.6) satisfies

$$
\left\|u_{N}\right\|_{L^{\infty}\left(L^{2}\right)}+\nu^{1 / 2}\left\|u_{N}\right\|_{L^{2}\left(H^{1}\right)}+\epsilon_{N}^{1 / 2}\left\|u_{N}\right\|_{L^{2}\left(H^{\alpha}\right)} \lesssim c .
$$

Proof. The argument is standard. We take the scalar product of the momentum equation in (3.6) with $u_{N}$ and we integrate over $] 0, T[$. Observing that

$$
\epsilon_{N}\left\|u_{N}\right\|_{H^{\alpha}}^{2}=\epsilon_{N}\left\|u_{N}\right\|_{H^{\alpha}}^{2}=\epsilon_{N}\left(Q * u_{N}, u_{N}\right)+\epsilon_{N} \sum_{1 \leq|k|_{\infty}<N_{i}}|k|^{2 \alpha}\left|u_{k}\right|^{2}
$$

we need to estimate the last term in the above inequality. Using the fact that $\epsilon_{N}=N_{i}^{-2 \alpha}$ and that $|k| \leq \sqrt{3}|k|_{\infty}$, we have

$$
\epsilon_{N} \sum_{1 \leq|k|_{\infty}<N_{i}}|k|^{2 \alpha}\left|u_{k}\right|^{2} \leq 3^{\alpha} N_{i}^{-2 \alpha} N_{i}^{2 \alpha} \sum_{1 \leq|k|_{\infty}<N_{i}}\left|u_{k}\right|^{2} \leq 3^{\alpha}\left\|u_{N}\right\|_{L^{2}}^{2}
$$

As a result, since $u_{N}$ is solenoidal (see Rem. 3.3) and using the above bound, we obtain

$$
\left\|u_{N}\right\|_{L^{2}}^{2}+\nu\left\|u_{N}\right\|_{L^{2}\left(H^{1}\right)}^{2}+\epsilon_{N}\left\|u_{N}\right\|_{L^{2}\left(H^{\alpha}\right)}^{2} \lesssim\left\|u_{0}\right\|_{L^{2}}+\frac{1}{2}\|f\|_{L^{2}\left(L^{2}\right)}^{2}+\left(3^{\alpha}+\frac{1}{2}\right)\left\|u_{N}\right\|_{L^{2}\left(L^{2}\right)}^{2} .
$$

The result is a consequence of Gronwall's lemma.

Remark 4.1. The proof of Lemma 4.1 suggests that instead of choosing $\epsilon_{N}=N_{i}^{-2 \alpha}$ we could have set $\epsilon_{N}=\frac{3^{1-\alpha}}{2} \nu N_{i}^{2-2 \alpha}$ since this choice yields

$$
\epsilon_{N} \sum_{1 \leq|k|_{\infty}<N_{i}}|k|^{2 \alpha}\left|u_{k}\right|^{2} \leq \frac{1}{2} \nu N_{i}^{2-2 \alpha} N_{i}^{2 \alpha-2} \sum_{1 \leq|k|_{\infty}<N_{i}}|k|^{2}\left|u_{k}\right|^{2} \leq \frac{1}{2} \nu\left\|u_{N}\right\|_{H^{1}}^{2}
$$

As a result, we would have

$$
\frac{1}{2} \nu\left\|u_{N}\right\|_{H^{1}}^{2}+\epsilon_{N}\left\|u_{N}\right\|_{H^{\alpha}}^{2} \leq \nu\left\|u_{N}\right\|_{H^{1}}^{2}+\epsilon_{N}\left(Q * u_{N}, u_{N}\right)
$$

and the Gronwall argument used at the end of the proof of Lemma 4.1 would apply equally well. We chose not to use this definition since it involves the viscosity and would imply that the dimension of the attractor of (3.6) depends on $\nu$, an undesirable feature for a LES technique. Moreover, the fact that $\epsilon_{N}$ does not depend on $\nu$ suggests that some parts of the argumentation developed in this paper could apply also to the incompressible Euler equations.

Lemma 4.2. Under the assumptions of Lemma 4.1, it holds

$$
\left\|u_{N}\right\|_{L^{p}\left(H^{2 / p}\right)}+\left\|u_{N}\right\|_{L^{p}\left(L^{q}\right)} \lesssim c, \quad \text { with } \quad \frac{3}{q}+\frac{2}{p}=\frac{3}{2}, \quad 2 \leq p, 2 \leq q \leq 6 .
$$

Proof. This result is standard and is a consequence of the interpolation inequality (see e.g. [9, p. 208]), $\|v\|_{H^{2 / p}} \lesssim$ $\|v\|_{L^{2}}^{1-2 / p}\|v\|_{H^{1}}^{2 / p}$, when $2 \leq p$, and the (compact) embedding $H^{2 / p}(\Omega) \subset L^{q}(\Omega)$ for $1 / q=1 / 2-2 /(3 p)$. 
Lemma 4.3. Under the assumptions of Lemma 4.1, the solution to (3.6) satisfies

$$
\left\|p_{N}\right\|_{L^{4 / 3}\left(L^{2}\right)} \lesssim c
$$

Proof. First, we observe that $\Delta: M_{N} \longrightarrow M_{N}$ is bijective. Then, we multiply the momentum equation in (3.6) by $\nabla \Delta^{-1} p_{N}$ (note that $\nabla \Delta^{-1} p_{N} \in X_{N}$ is an admissible test function). By using several integrations by parts, we obtain

$$
\begin{aligned}
\left\|p_{N}\right\|_{L^{2}}^{2} & =\left(\nabla p_{N}, \nabla \Delta^{-1} p_{N}\right) \\
& =\left(-\partial_{t} u_{N}+\nu \Delta u_{N}-u_{N} \cdot \nabla u_{N}+f, \nabla \Delta^{-1} p_{N}\right) \\
& =-\left(u_{N} \cdot \nabla u_{N}, \nabla \Delta^{-1} p_{N}\right), \quad \text { since } u_{N} \text { and } f \text { are solenoidal } \\
& =-\left(\nabla \cdot\left(u_{N} \otimes u_{N}\right), \nabla \Delta^{-1} p_{N}\right) \\
& =\left(u_{N} \otimes u_{N}, \nabla \nabla \Delta^{-1} p_{N}\right) \\
& \lesssim\left\|u_{N}\right\|_{L^{4}}^{2}\left\|p_{N}\right\|_{L^{2}} .
\end{aligned}
$$

As a result $\left\|p_{N}\right\|_{L^{2}} \lesssim\left\|u_{N}\right\|_{L^{4}}^{2}$. The conclusion is a consequence of Lemma 4.2 with $q=4$ and $p=\frac{8}{3}$.

Lemma 4.4. Let $s$ be a real number such that $s \geq 2 \alpha-1>\frac{3}{2}$. Under the assumptions of Lemma 4.1, the solution to (3.6) satisfies

$$
\left\|\partial_{t} u_{N}\right\|_{L^{4 / 3}\left(H^{-s}\right)} \lesssim c
$$

Proof. Since $P_{N} \in \mathcal{L}\left(H^{s}(\Omega) ; H^{s}(\Omega)\right)$ (see Lem. 3.1), for all $v \in H^{s}(\Omega)$, we have

$$
\begin{aligned}
\epsilon_{N}\left(Q * u_{N}, P_{N} v\right) & \lesssim\left\|u_{N}\right\|_{H^{1}}\left\|P_{N} v\right\|_{H^{2 \alpha-1}} \lesssim\left\|u_{N}\right\|_{H^{1}}\|v\|_{H^{s}}, \\
\nu\left(\nabla u_{N}, \nabla P_{N} v\right) & \lesssim\left\|u_{N}\right\|_{H^{1}}\left\|P_{N} v\right\|_{H^{1}} \lesssim\left\|u_{N}\right\|_{H^{1}}\|v\|_{H^{s}}, \\
\left(u_{N} \cdot \nabla u_{N}, P_{N} v\right) & \lesssim\left\|u_{N}\right\|_{L^{2}}\left\|u_{N}\right\|_{H^{1}}\left\|P_{N} v\right\|_{L^{\infty}} \lesssim\left\|u_{N}\right\|_{H^{1}}\|v\|_{H^{s}}, \quad s>\frac{3}{2}, \\
\left(p_{N}, \nabla \cdot P_{N} v\right) & \lesssim\left\|p_{N}\right\|_{L^{2}}\left\|P_{N} v\right\|_{H^{1}} \lesssim\left\|p_{N}\right\|_{L^{2}}\|v\|_{H^{s}}, \\
\left(f, P_{N} v\right) & \lesssim\|f\|_{L^{2}}\left\|P_{N} v\right\|_{L^{2}} \lesssim\|f\|_{L^{2}}\|v\|_{H^{s}} .
\end{aligned}
$$

Now, observing that $\left(\partial_{t} u_{N}, v\right)=\left(\partial_{t} u_{N}, P_{N} v\right)$ for all $v \in H^{s}(\Omega)$, and using the discrete momentum equation, we obtain (provided $v \neq 0$ )

$$
\frac{\left(\partial_{t} u_{N}, v\right)}{\|v\|_{H^{s}}} \lesssim\|f\|_{L^{2}}+\left\|u_{N}\right\|_{H^{1}}+\left\|p_{N}\right\|_{L^{2}}
$$

The final statement is a consequence of Lemma 4.1 and Lemma 4.3.

\subsection{Proof of Theorem 4.1}

We briefly outline the main steps of the proof for the arguments are quite standard.

Since $u_{N}$ is bounded uniformly in $L^{p}\left(0, T ; H^{2 / p}(\Omega)\right)$, such that $2 \leq p<\infty$ and $\partial_{t} u_{N}$ is bounded uniformly in $L^{4 / 3}\left(0, T ; H^{-s}(\Omega)\right)$, such that $s \geq 2 \alpha-1$, we infer from Aubin-Lions compacity lemma (see Lions [24, p. 57]) that there exists a subsequence $\left(u_{N_{l}}\right)$ converging weakly in $L^{2}\left(0, T ; H^{1}(\Omega)\right)$ and strongly in any $L^{p}\left(0, T ; L^{q}(\Omega)\right)$, such that $2 \leq p<\frac{4 q}{3(q-2)}$, and that $\left(\partial_{t} u_{N_{l}}\right)$ converges weakly in $L^{4 / 3}\left(0, T ; H^{-s}(\Omega)\right)$. Moreover, since $\left(p_{N}\right)$ is bounded uniformly in $L^{4 / 3}\left(0, T ; L^{2}(\Omega)\right)$, there exists a subsequence $\left(p_{N_{l}}\right)$ converging weakly in $L^{4 / 3}\left(0, T ; L^{2}(\Omega)\right)$. Let $u$ and $p$ denote these limits, and let $v$ be a test function in $L^{4}\left(0, T ; H^{s}(\Omega)\right)$. Then

1) $\int_{Q_{T}} \partial_{t} u_{N} \cdot v \rightarrow \int_{Q_{T}} \partial_{t} u \cdot v$, since $\partial_{t} u_{N} \rightarrow \partial_{t} u$ in $L^{4 / 3}\left(H^{-s}\right)$.

2) $\int_{Q_{T}} \nabla u_{N}: \nabla v \rightarrow \int_{Q_{T}} \nabla u: \nabla v$, since $\nabla v \in L^{4}\left(0, T ; H^{s-1}\right)$ and $\nabla u_{N} \rightarrow \nabla u$ in $L^{2}\left(L^{2}\right) \subset L^{4 / 3}\left(H^{-s+1}\right)$. 
3) $\int_{Q_{T}} p_{N} \nabla \cdot v \rightarrow \int_{Q_{T}} p \nabla \cdot v$, since $p_{N} \rightarrow p$ in $L^{4 / 3}\left(L^{2}\right)$ and $\nabla \cdot v \in L^{4}\left(H^{s-1}\right) \subset L^{4 / 3}\left(L^{2}\right)$.

4) $\left|\epsilon_{N} \int_{Q_{T}} v \cdot Q * u_{N}\right| \lesssim \epsilon_{N} \int_{0}^{T}\left\|u_{N}\right\|_{H^{1}}\|v\|_{H^{s}} \lesssim \epsilon_{N}\left\|u_{N}\right\|_{L^{2}\left(H^{1}\right)}\|v\|_{L^{2}\left(H^{s}\right)} \rightarrow 0$

5) Since $u_{N} \rightarrow u$ in $L^{4}\left(L^{2}\right)$ and $v \in L^{4}\left(H^{s}\right) \subset L^{4}\left(L^{\infty}\right), s>3 / 2$, we infer that $v \otimes u_{N} \rightarrow v \otimes u$ in $L^{2}\left(L^{2}\right)$. Hence, $\int_{Q_{T}} v \otimes u_{N}: \nabla u_{N} \rightarrow \int_{Q_{T}} v \otimes u: \nabla u$ since $\nabla u_{N} \rightarrow \nabla u$ in $L^{2}\left(L^{2}\right)$.

Finally $P_{N} u_{0}=u_{N}(0) \rightarrow u(0)$ in $H^{-s}(\Omega)$; hence, $u(0)=u_{0}$. The theorem is proved.

\section{Convergence to A Dissipative solution of the nse}

In this section, we show that the spectral hyperviscosity model (3.6) guarantees convergence, as $N$ goes to infinity, to a dissipative solution of the Navier-Stokes equation (in the sense of Def. 1.1). This result is stated in the following theorem.

Theorem 5.1. Let $f \in L^{2}\left(0, T ; L^{2}(\Omega)\right)$ and $u_{0} \in H^{\alpha}(\Omega)$. Assuming that (3.2), (3.4), and (3.3) hold, the solution $u_{N}$ to (3.6) is such that subsequences of $\left(u_{N}\right)$ converge weakly in $L^{2}\left(0, T ; H^{1}\right)$ and strongly in any $L^{p}\left(0, T ; L^{q}\right)$ with $2 \leq p<\frac{4 q}{3(q-2)}<+\infty$, to a dissipative solution of (1.1) as $N$ goes to infinity.

\subsection{Preliminaries}

Before proving Theorem 5.1, we establish some technical results. Let us define the following distributions

$$
\begin{aligned}
Q_{h}(x) & =(2 \pi)^{-3} \sum_{|k|_{\infty}<N_{i}}|k|^{2 \alpha} \mathrm{e}^{\mathrm{i} k \cdot x}, \\
Q_{t}(x) & =(2 \pi)^{-3} \sum_{N<|k|_{\infty}}|k|^{2 \alpha} \mathrm{e}^{\mathrm{i} k \cdot x}, \\
S(x) & =(2 \pi)^{-3} \sum_{|k|_{\infty} \geq 0}|k|^{\alpha} \mathrm{e}^{\mathrm{i} k \cdot x} .
\end{aligned}
$$

Let us set $\tilde{Q}=Q+Q_{h}+Q_{t}$. It is clear that $Q_{t} * v_{N}=0$ for all $v_{N}$ in $X_{N}$. Furthermore, we have $S * S=\tilde{Q}$, that is, $(\tilde{Q} * v, w)=(S * v, S * w)$ for any sufficiently smooth functions $v$ and $w$.

Lemma 5.1. Let $0<\gamma<2 \alpha$, then $\forall u \in H^{\gamma}(\Omega), \forall v \in L^{2}(\Omega), \forall \phi \in L^{\infty}(\Omega)$, the following bound holds:

$$
\epsilon_{N}\left|\left(Q_{h} * u, v \phi\right)\right| \lesssim N^{-\theta \gamma}\|u\|_{H^{\gamma}}\|v\|_{L^{2}}\|\phi\|_{L^{\infty}} .
$$

Proof. From Hölder's inequality, we immediately obtain:

$$
\epsilon_{N}\left|\left(Q_{h} * u, v \phi\right)\right| \leq \epsilon_{N}\left\|Q_{h} * u\right\|_{L^{2}}\|v\|_{L^{2}}\|\phi\|_{L^{\infty}} .
$$

From the definition of $Q_{h}$ we derive

$$
\begin{aligned}
\epsilon_{N}^{2}\left\|Q_{h} * u\right\|_{L^{2}}^{2} & \lesssim \epsilon_{N}^{2} \sum_{|k|_{\infty}<N_{i}}|k|^{4 \alpha}\left|u_{k}\right|^{2} \\
& \lesssim N_{i}^{-4 \alpha} N_{i}^{4 \alpha-2 \gamma} \sum_{|k|_{\infty}<N_{i}}|k|^{2 \gamma}\left|u_{k}\right|^{2} \\
& \lesssim N_{i}^{-2 \gamma}\|u\|_{H^{\gamma}}^{2}=N^{-2 \theta \gamma}\|u\|_{H^{\gamma}}^{2}
\end{aligned}
$$

The conclusion is now straightforward. 
Lemma 5.2. Let $s$ be a real number such that $s>\frac{3}{2}+\alpha$. The following estimate holds for all $u \in H^{\alpha-1}(\Omega)$ and all $\phi \in H^{s}(\Omega)$,

$$
\|S *(u \phi)-(S * u) \phi\|_{L^{2}} \lesssim\|u\|_{H^{\alpha-1}}\|\phi\|_{H^{s}} .
$$

Proof. First, it is clear that

$$
\begin{aligned}
& S *(u \phi)=\sum_{k \in \mathbb{Z}^{3}}|k|^{\alpha} \sum_{l+l^{\prime}=k} u_{l} \phi_{l^{\prime}} \mathrm{e}^{\mathrm{i} k \cdot x}, \\
& (S * u) \phi=\sum_{l \in \mathbb{Z}^{3}} \sum_{l^{\prime} \in \mathbb{Z}^{3}}|l|^{\alpha} u_{l} \phi_{l^{\prime}} \mathrm{e}^{\mathrm{i}\left(l+l^{\prime}\right) \cdot x} .
\end{aligned}
$$

Then, it follows that

$$
\begin{aligned}
\|S *(u \phi)-(S * u) \phi\|_{L^{2}}^{2} & =\sum_{k \in \mathbb{Z}^{3}}\left(\sum_{l+l^{\prime}=k}\left(|k|^{\alpha}-|l|^{\alpha}\right) u_{l} \phi_{l^{\prime}}\right)^{2} \\
& \leq \sum_{k \in \mathbb{Z}^{3}}\left(\sum_{l+l^{\prime}=k, l^{\prime} \neq 0}\left|l^{\prime}\right|^{2 s} \phi_{l^{\prime}}^{2}\right)\left(\sum_{l+l^{\prime}=k, l^{\prime} \neq 0} \frac{\left(|k|^{\alpha}-|l|^{\alpha}\right)^{2}}{\left|l^{\prime}\right|^{2 s}} u_{l}^{2}\right) \\
& \lesssim\|\phi\|_{H^{s}}^{2} \sum_{k \in \mathbb{Z}^{3}} \sum_{l \neq k, l \in \mathbb{Z}^{3}} \frac{\left(|k|^{\alpha}-|l|^{\alpha}\right)^{2}}{|k-l|^{2 s}} u_{l}^{2} \\
& \lesssim\|\phi\|_{H^{s}}^{2} \sum_{l \in \mathbb{Z}^{3}}\left(1+|l|^{2}\right)^{\alpha-1} u_{l}^{2} \sum_{l \neq k, k \in \mathbb{Z}^{3}} \frac{\left(|k|^{\alpha}-|l|^{\alpha}\right)^{2}}{|k-l|^{2 s}\left(1+|l|^{2}\right)^{\alpha-1}}
\end{aligned}
$$

Let us denote by $I(l)$ the second series in the last right-hand side.

$$
I(l)=\sum_{l \neq k, k \in \mathbb{Z}^{3}} \frac{\left(|k|^{\alpha}-|l|^{\alpha}\right)^{2}}{|k-l|^{2 s}\left(1+|l|^{2}\right)^{\alpha-1}}=\sum_{k \neq 0, k \in \mathbb{Z}^{3}} \frac{\left(|k+l|^{\alpha}-|l|^{\alpha}\right)^{2}}{|k|^{2 s}\left(1+|l|^{2}\right)^{\alpha-1}} .
$$

Inspection reveals that this series is absolutely convergent if $2 s>3+2 \alpha$ and bounded uniformly with respect to $l$. Then, the conclusion follows easily.

Lemma 5.3. Let $s$ be a real number such that $s>\frac{3}{2}+\alpha$. The following estimate holds for all $u \in H^{\alpha}(\Omega)$ and all $\phi \in H^{s}(\Omega)$,

$$
\|u \phi\|_{H^{\alpha}} \lesssim\|u\|_{H^{\alpha}}\|\phi\|_{H^{s}} .
$$

Proof. The proof is similar to that of Lemma 5.2.

\subsection{Proof of Theorem $\mathbf{5 . 1}$}

Let $\phi$ be a non negative function infinitely differentiable and compactly supported in $(0, T)$ (or at least in $\mathcal{D}\left(0, T ; H^{s}(\Omega)\right)$ with $\left.s>\frac{3}{2}+\alpha\right)$. Testing the momentum equation in (3.6) by $P_{N}\left(u_{N} \phi\right)$, we obtain:

$$
\begin{aligned}
\left(\partial_{t} u_{N}, P_{N}\left(u_{N} \phi\right)\right)+\left(u_{N} \cdot \nabla u_{N}, P_{N}\left(u_{N} \phi\right)\right)-\left(p_{N},\right. & \left.\nabla \cdot P_{N}\left(u_{N} \phi\right)\right) \\
& +\nu\left(\nabla u_{N}, \nabla P_{N}\left(u_{N} \phi\right)\right)+\epsilon_{N}\left(Q * u_{N}, P_{N}\left(u_{N} \phi\right)\right)=\left(f, P_{N}\left(u_{N} \phi\right)\right)
\end{aligned}
$$


Each of the terms on the left-hand side of the above equation are now treated separately in the following steps:

Step 1. We have $\int_{0}^{T}\left(\partial_{t} u_{N}, P_{N}\left(u_{N} \phi\right)\right)=\int_{0}^{T}\left(\partial_{t} u_{N}, u_{N} \phi\right)=-\frac{1}{2} \int_{0}^{T}\left(u_{N}^{2}, \partial_{t} \phi\right)$ and the limit is $-\frac{1}{2} \int_{0}^{T}\left(u^{2}, \partial_{t} \phi\right)$ since $u_{N}^{2} \rightarrow u^{2}$ in $L^{p}\left(L^{1}\right)$ for any $2 \leq p<\infty$.

Step 2. Since $P_{N}$ commutes with differentiation operators, the viscous term yields $\int_{0}^{T}\left(\nabla u_{N}, \nabla P_{N}\left(u_{N} \phi\right)\right)=$ $\int_{0}^{T}\left(\nabla u_{N}, \nabla\left(u_{N} \phi\right)\right)=\int_{0}^{T}\left(\left|\nabla u_{N}\right|^{2}, \phi\right)-\left(\frac{1}{2}\left|u_{N}\right|^{2}, \Delta \phi\right)$. The second term clearly converges to $-\left(\frac{1}{2}|u|^{2}, \Delta \phi\right)$ since $u_{N}^{2} \rightarrow u^{2}$ in $L^{p}\left(L^{1}\right)$ for any $2 \leq p<\infty$. For the other term we proceed as follows:

$$
\begin{aligned}
\int_{0}^{T}\left(\left|\nabla u_{N}\right|^{2}, \phi\right) & =\int_{0}^{T}\left(\left|\nabla\left(u_{N}-u+u\right)\right|^{2}, \phi\right) \\
& =\int_{0}^{T}\left(\left|\nabla\left(u_{N}-u\right)\right|^{2}+2 \nabla\left(u_{N}-u\right): \nabla u+|\nabla u|^{2}, \phi\right) .
\end{aligned}
$$

The second term in the right-hand side goes to zero since $u_{N} \rightarrow u$ in $L^{2}\left(H^{1}\right)$. As a result $\liminf \int_{0}^{T}\left(\left|\nabla u_{N}\right|^{2}, \phi\right) \geq$ $\int_{0}^{T}\left(|\nabla u|^{2}, \phi\right)$. That is,

$$
\liminf _{N \rightarrow+\infty} \int_{0}^{T}\left(\nabla u_{N}, \nabla P_{N}\left(u_{N} \phi\right)\right) \geq \int_{0}^{T}\left(|\nabla u|^{2}, \phi\right)-\left(\frac{1}{2}|u|^{2}, \Delta \phi\right) .
$$

Step 3. Again, owing to the commutation of $P_{N}$ with differentiation operators, and because $u_{N}$ is solenoidal, we have for the pressure term $\int_{0}^{T}\left(p_{N}, \nabla \cdot\left(P_{N}\left(u_{N} \phi\right)\right)\right)=\int_{0}^{T}\left(p_{N}, \nabla \cdot\left(u_{N} \phi\right)\right)=\int_{0}^{T}\left(p_{N} u_{N}, \nabla \phi\right)$. As a result,

$$
\int_{0}^{T}\left(p_{N}, \nabla \cdot\left(P_{N}\left(u_{N} \phi\right)\right)\right) \rightarrow \int_{0}^{T}(p u, \nabla \phi)
$$

since $p_{N} \rightarrow p$ in $L^{4 / 3}\left(L^{2}\right)$ and $u_{N} \nabla \phi \rightarrow u \nabla \phi$ in $L^{4}\left(L^{2}\right)$.

Step 4. For the hyperviscosity term we proceed as follows. Since $Q * u_{N} \in X_{N}$, we have

$$
\begin{aligned}
\epsilon_{N}\left(Q * u_{N}, P_{N}\left(u_{N} \phi\right)\right) & =\epsilon_{N}\left(Q * u_{N}, u_{N} \phi\right), \\
& =\epsilon_{N}\left(\left(\tilde{Q}-Q_{h}-Q_{t}\right) * u_{N}, u_{N} \phi\right), \\
& =\epsilon_{N}\left(\tilde{Q} * u_{N}, u_{N} \phi\right)-\epsilon_{N}\left(Q_{h} * u_{N}, u_{N} \phi\right), \\
& =\epsilon_{N}\left(S * u_{N}, S *\left(u_{N} \phi\right)\right)-\epsilon_{N}\left(Q_{h} * u_{N}, u_{N} \phi\right), \\
& =\epsilon_{N}\left(\left|S * u_{N}\right|^{2}, \phi\right)+R_{1}+R_{2}
\end{aligned}
$$

where the two remainders are defined as $R_{1}=\epsilon_{N}\left(S * u_{N}, S *\left(u_{N} \phi\right)-\left(S * u_{N}\right) \phi\right)$ and $R_{2}=-\epsilon_{N}\left(Q_{h} * u_{N}, u_{N} \phi\right)$. Then

$$
\begin{aligned}
\left|R_{1}\right| & \leq \epsilon_{N}\left\|S * u_{N}\right\|_{L^{2}}\left\|S *\left(u_{N} \phi\right)-\left(S * u_{N}\right) \phi\right\|_{L^{2}}, \\
& \lesssim \epsilon_{N}\left\|u_{N}\right\|_{H^{\alpha}}\left\|u_{N}\right\|_{H^{\alpha-1}}\|\phi\|_{H^{s}}, \\
& \lesssim \epsilon_{N}\left\|u_{N}\right\| H_{H^{\alpha}}\left\|u_{N}\right\|_{L^{2}}^{1-\gamma}\left\|u_{N}\right\|_{H^{\alpha}}^{\gamma}\|\phi\|_{H^{s}}, \\
& \lesssim \epsilon_{N}^{\frac{1}{2 \alpha}}\left(\epsilon_{N}^{\frac{1}{2}}\left\|u_{N}\right\|_{H^{\alpha}}\right)^{2-\frac{1}{\alpha}}\|\phi\|_{H^{s}}, \\
& \lesssim \epsilon_{N}^{\frac{1}{2 \alpha}}\left(\epsilon_{N}\left\|u_{N}\right\|_{H^{\alpha}}^{2}+\|\phi\|_{H^{s}}^{2 \alpha}\right) .
\end{aligned}
$$

See Lemma $5.2, s>\frac{3}{2}+\alpha$,

Interp. ineq., $\gamma=\frac{\alpha-1}{\alpha}$, 
Then, it is clear that $\int_{0}^{T}\left|R_{1}\right| \rightarrow 0$ as $N \rightarrow+\infty$. Concerning the second remainder we use Lemma 5.1 with $\gamma=1$. Then

$$
\int_{0}^{T}\left|R_{2}\right| \lesssim N^{-\theta}\|\phi\|_{L^{\infty}\left(L^{\infty}\right)} \int_{0}^{T}\left(\left\|u_{N}\right\|_{H^{1}}^{2}+\left\|u_{N}\right\|_{L^{2}}^{2}\right)
$$

so that $\int_{0}^{T}\left|R_{2}\right| \rightarrow 0$ as $N \rightarrow+\infty$. As a result, $\liminf \int_{0}^{T} \epsilon_{N}\left(Q * u_{N}, P_{N}\left(u_{N} \phi\right)\right) \geq 0$.

Step 5. Unsurprisingly, the treatment of the nonlinear term poses the greatest difficulties. We make here full use of the hypotheses on $\alpha$ and $\epsilon_{N}$ in (3.2) and (3.4). Defining $R=\left(u_{N} \cdot \nabla u_{N}, P_{N}\left(u_{N} \phi\right)-u_{N} \phi\right)$ and because $u_{N}$ is solenoidal, we have

$$
\left(u_{N} \cdot \nabla u_{N}, P_{N}\left(u_{N} \phi\right)\right)=\left(u_{N} \cdot \nabla u_{N}, u_{N} \phi\right)+R=-\left(\frac{1}{2}\left|u_{N}\right|^{2} u_{N}, \nabla \phi\right)+R
$$

Since $u_{N} \rightarrow u$ in $L^{4-\delta}\left(L^{3}\right)$, for all $0<\delta<1$, we infer $\left|u_{N}\right|^{2} u_{N} \rightarrow|u|^{2} u$ in $L^{\frac{4-\delta}{3}}\left(L^{1}\right)$. As a result,

$$
-\int_{0}^{T}\left(\frac{1}{2}\left|u_{N}\right|^{2} u_{N}, \nabla \phi\right) \rightarrow-\int_{0}^{T}\left(\frac{1}{2}|u|^{2} u, \nabla \phi\right), \quad \text { as } N \rightarrow+\infty .
$$

For the remainder $R$ we have

$$
\begin{aligned}
|R| & =\left|\left(u_{N} \cdot \nabla u_{N}, P_{N}\left(u_{N} \phi\right)-u_{N} \phi\right)\right|, \\
& \lesssim\left\|u_{N}\right\|_{L^{4}}^{2}\left\|\nabla\left(P_{N}\left(u_{N} \phi\right)-u_{N} \phi\right)\right\|_{L^{2}}, \\
& \lesssim N^{1-\alpha}\left\|u_{N}\right\|_{L^{4}}^{2}\left\|u_{N} \phi\right\|_{H^{\alpha}}, \\
& \lesssim N^{1-\alpha}\left\|u_{N}\right\|_{L^{4}}^{2}\left\|u_{N}\right\|_{H^{\alpha}}\|\phi\|_{H^{s}},
\end{aligned}
$$

Approximation property,

Lemma 5.3.

To bound $\left\|u_{N}\right\|_{L^{4}}$ we proceed as follows.

$$
\begin{aligned}
\left\|u_{N}\right\|_{L^{4}}^{2} & \lesssim\left\|u_{N}\right\|_{H^{r}}^{2}, \\
& \lesssim\left\|u_{N}\right\|_{L^{2}}^{2(1-\gamma)}\left\|u_{N}\right\|_{H^{\alpha}}^{2 \gamma} \\
& \lesssim\left\|u_{N}\right\|_{H^{\alpha}}^{\frac{3}{2 \alpha}}
\end{aligned}
$$

At this point, there are two possibilities: either $\frac{3}{2 \alpha} \leq 1$ or $\frac{3}{2 \alpha}>1$.

If $\alpha<\frac{3}{2}$, then

$$
\begin{aligned}
\left\|u_{N}\right\|_{L^{4}}^{2} & \lesssim\left\|u_{N}\right\|_{H^{\alpha}}\left\|u_{N}\right\|_{H^{\alpha}}^{\frac{3}{2 \alpha}-1} \\
& \lesssim N^{\frac{3}{2}-\alpha}\left\|u_{N}\right\|_{H^{\alpha}}\left\|u_{N}\right\|_{L^{2}}^{\frac{3}{2 \alpha}-1},
\end{aligned}
$$

Then, it is clear that

$$
|R| \lesssim N^{\frac{5}{2}-2 \alpha}\left\|u_{N}\right\|_{H^{\alpha}}^{2}\|\phi\|_{H^{s}}=N^{\frac{5}{2}-2 \alpha+\beta} \epsilon_{N}\left\|u_{N}\right\|_{H^{\alpha}}^{2}\|\phi\|_{H^{s}} .
$$

That is to say, owing to the hypotheses (3.2) and (3.4), we have $\int_{0}^{T}|R| \rightarrow 0$ as $N \rightarrow+\infty$. 
On the other hand, if $\alpha \geq \frac{3}{2}$, then

$$
\begin{aligned}
|R| & \lesssim N^{1-\alpha}\left\|u_{N}\right\|_{H^{\alpha}}^{1+\frac{3}{2 \alpha}}\|\phi\|_{H^{s}}, & \\
& \lesssim N^{1-\alpha+\frac{\beta}{2}\left(1+\frac{3}{2 \alpha}\right)}\left(\epsilon_{N}\left\|u_{N}\right\|_{H^{\alpha}}^{2}\right)^{\frac{1}{2}\left(1+\frac{3}{2 \alpha}\right)}\|\phi\|_{H^{s}}, & \\
& \lesssim N^{1-\alpha+\frac{\beta}{2}\left(1+\frac{3}{2 \alpha}\right)}\left(\epsilon_{N}\left\|u_{N}\right\|_{H^{\alpha}}^{2}+\|\phi\|_{H^{s}}^{r}\right), & \frac{1}{r}+\frac{3+2 \alpha}{4 \alpha}=1 .
\end{aligned}
$$

Then again, owing to the hypothesis (3.4), it is clear that $\int_{0}^{T}|R| \rightarrow 0$ as $N \rightarrow+\infty$. The theorem is proved.

\section{Concluding Remarks}

We have proposed in this paper a spectral hyperviscosity model that regularizes the Navier-Stokes equations, provides solutions that converge to dissipative weak solutions of the Navier-Stokes equations when the cutoff goes to infinity, and preserves spectral accuracy for smooth solutions. To the best of our knowledge, none of the existing LES spectral techniques satisfy more than one of these properties. Numerical experiments will be initiated to validate these theoretical results and to compare the behavior of the spectral hyperviscosity model with other existing LES spectral techniques.

The model proposed here may seem to be crude when compared with those currently proposed in the turbulence literature. In particular, the model does not account for the so-called back-scatter effect. Further work is certainly necessary to understand the mathematical implications of such a phenomenon.

Another annoying issue is that the present discussion is restricted to periodic boundary conditions, whereas most engineering applications targeted by LES involve complex geometry where the periodicity hypothesis does not hold. We are currently extending the present work to account for this major objection, in particular by working with finite elements. The major obstacle in the way is that the nice pressure estimate of Lemma 4.3 is no longer available. This estimate must be replaced by estimates in $L^{\frac{5}{4}}\left(L^{\frac{5}{3}}\right)$ which seem to be technically difficult to obtain with finite elements. We refer the interested reader to the Appendix of the paper of Caffarelli, Kohn, and Nirenberg [4].

Acknowledgements. The first author gratefully acknowledges the support of CNRS and of the Texas Institute for Computational and Applied Mathematics under a TICAM Visiting Faculty Fellowship.

\section{REFERENCES}

[1] N.A. Adams and S. Stolz, A subgrid-scale deconvolution approach for shock capturing. J. Comput. Phys. 178 (2002) $391-426$.

[2] C. Basdevant, B. Legras, R. Sadourny and M. Béland, A study of barotropic model flows: intermittency, waves and predictability. J. Atmospheric Sci. 38 (1981) 2305-2326.

[3] H. Brezis, Analyse Fonctionnelle, Théorie et Applications. Masson, Paris (1983).

[4] L. Caffarelli, R. Kohn and L. Nirenberg, Partial regularity of suitable weak solutions of the Navier-Stokes equations. Comm. Pure Appl. Math. 35 (1982) 771-831.

[5] G.-Q. Chen, Q. Du and E. Tadmor, Spectral viscosity approximations to multidimensionnal scalar conservation laws. Math. Comp. 61 (1993) 629-643.

[6] S. Chen, C. Foias, D.D. Holm, E. Olson, E.S. Titi and S. Wynne, A connection between the Camassa-Holm equation and turbulent flows in channels and pipes. Phys. Fluids 11 (1999) 2343-2353.

[7] J.P. Chollet and M. Lesieur, Parametrization of small scales of three-dimensional isotropic turbulence utilizing spectral closures. J. Atmospheric Sci. 38 (1981) 2747-2757.

[8] G.-H. Cottet, D. Jiroveanu and B. Michaux, Vorticity dynamics and turbulence models for Large-Eddy Simulations. ESAIM: M2AN 37 (2003) 187-207.

[9] C.R. Doering and J.D. Gibbon, Applied analysis of the Navier-Stokes equations. Cambridge texts in applied mathematics, Cambridge University Press (1995).

[10] J. Duchon and R. Robert, Inertial energy dissipation for weak solutions of incompressible Euler and Navier-Stokes equations. Nonlinearity 13 (2000) 249-255. 
[11] J.-L. Guermond, J.T. Oden and S. Prudhomme, Mathematical perspectives on the Large Eddy Simulation models for turbulent flows. J. Math. Fluid Mech. (2003). In press.

[12] S. Kaniel, On the initial value problem for an incompressible fluid with nonlinear viscosity. J. Math. Mech. 19 (1970) 681-706.

[13] G.-S. Karamanos and G.E. Karniadakis, A spectral vanishing viscosity method for large-eddy simulations. J. Comput. Phys. $163(2000) 22-50$

[14] N.K.-R. Kevlahan and M. Farge, Vorticity filaments in two-dimensional turbulence: creation, stability and effect. J. Fluid Mech. 346 (1997) 49-76.

[15] R.H. Kraichnan, Eddy viscosity in two and three dimensions. J. Atmospheric Sci. 33 (1976) 1521-1536.

[16] O.A. Ladyženskaja, Modification of the Navier-Stokes equations for large velocity gradients, in Seminars in Mathematics V.A. Stheklov Mathematical Institute, Vol. 7, Boundary value problems of mathematical physics and related aspects of function theory, Part II, O.A. Ladyženskaja Ed., New York, London (1970). Consultant Bureau.

[17] O.A. Ladyženskaja, New equations for the description of motion of viscous incompressible fluds and solvability in the large of boundary value problems for them, in Proc. of the Stheklov Institute of Mathematics, number 102 (1967), Boundary value problems of mathematical physics, O.A. Ladyženskaja Ed., V, Providence, Rhode Island (1970). AMS.

[18] E. Lamballais, O. Métais and M. Lesieur, Spectral-dynamic model for large-eddy simulations of turbulent rotating channel flow. Theoret. Comput. Fluid Dynamics 12 (1998) 149-177.

[19] A. Leonard, Energy cascade in Large-Eddy simulations of turbulent fluid flows. Adv. Geophys. 18 (1974) $237-248$.

[20] J. Leray, Essai sur le mouvement d'un fluide visqueux emplissant l'espace. Acta Math. 63 (1934) 193-248.

[21] M. Lesieur and R. Roggalo, Large-eddy simulations of passive scalar diffusion in isotropic turbulence. Phys. Fluids A 1 (1989) $718-722$.

[22] J.-L. Lions, Quelques résultats d'existence dans des équations aux dérivées partielles non linéaires. Bull. Soc. Math. France 87 (1959) 245-273.

[23] J.-L. Lions, Sur certaines équations paraboliques non linéaires. Bull. Soc. Math. France 93 (1965) 155-175.

[24] J.-L. Lions, Quelques méthodes de résolution des problèmes aux limites non linéaires, Vol 1. Dunod, Paris (1969).

[25] Y. Maday, M. Ould Kaber and E. Tadmor, Legendre pseudospectral viscosity method for nonlinear conservation laws. SIAM J. Numer. Anal. 30 (1993) 321-342.

[26] D. McComb and A. Young, Explicit-scales projections of the partitioned non-linear term in direct numerical simulation of the Navier-Stokes equation, in Second Monte Verita Colloquium on Fundamental Problematic Issues in Fluid Turbulence, Ascona, March 23-27 (1998). Available on the Internet at http://xxx.soton.ac.uk/abs/physics/9806029.

[27] V. Scheffer, Hausdorff measure and the Navier-Stokes equations. Comm. Math. Phys. 55 (1977) 97-112.

[28] V. Scheffer, Nearly one-dimensional singularities of solutions to the Navier-Stokes inequality. Comm. Math. Phys. 110 (1987) $525-551$.

[29] J. Smagorinsky, General circulation experiments with the primitive equations, part i: the basic experiment. Monthly Wea. Rev. 91 (1963) 99-152.

[30] E. Tadmor, Convergence of spectral methods for nonlinear conservation laws. SIAM J. Numer. Anal. 26 (1989) 30-44.

To access this journal online:

www.edpsciences.org 